\title{
DISEASE RESISTANCE
}

\section{Safeguard by root microbiome}

Nat. Biotechnol. 36, 1100-1109 (2018).

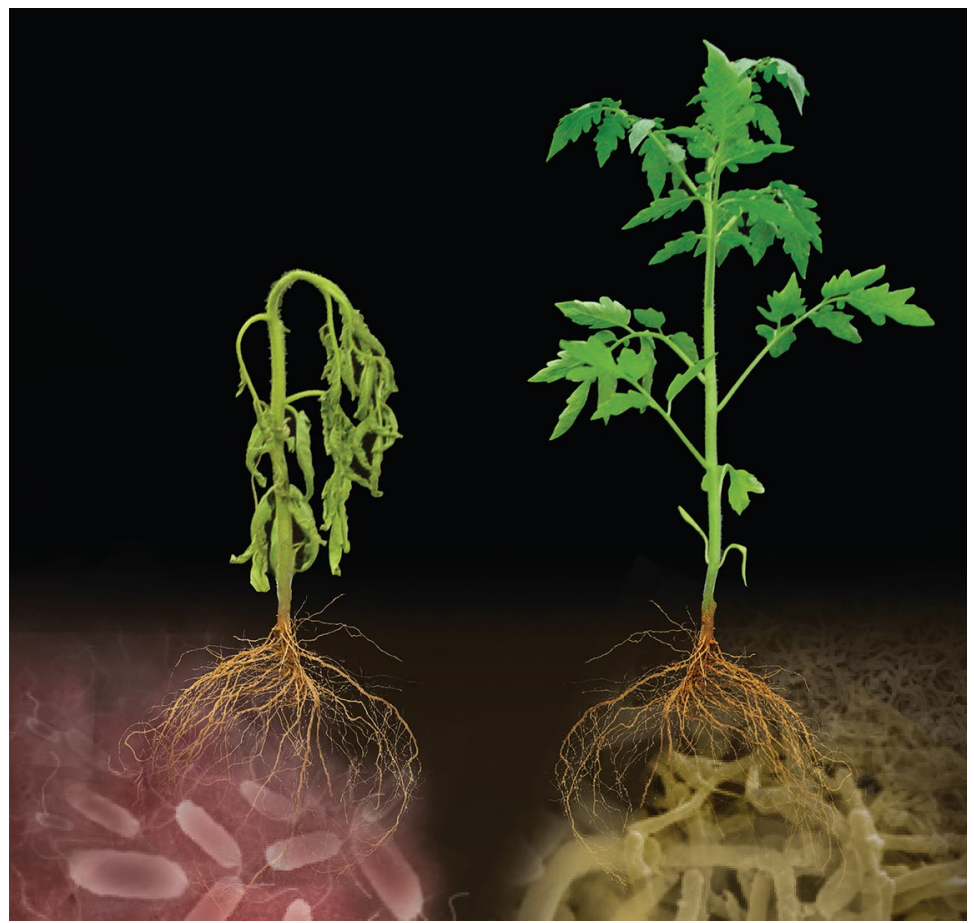

Credit: Courtesy of Irin M. Kim (Seoul Women's University), Jihyun F. Kim (Yonsei University) and Seon-Woo Lee (Dong-A University)

Plants have a sophisticated internal immune system, as well as an external rhizosphere ecosystem, that reacts to pathogens and determines disease development. While plant rhizosphere microorganisms have been known to be important for controlling diseases, little is known about their involvement in plant defence. To examine how the root microbiome contributes to disease resistance, Min-Jung Kwak, from Yonsei University, Republic of Korea, and colleagues, used the tomato plant and bacterial wilt as a model system.

Bacterial wilt of tomato is caused by the soil-borne pathogen, Ralstonia solanacearum (left in the figure). The tomato variety Hawaii 7996 is highly resistant to the bacterium, while the variety Moneymaker is susceptible. The researchers analysed the root microbiomes of both varieties in an outdoor mesocosm plastic house and in growth-chamber pots. Amplicon sequencing of the $16 \mathrm{~S}$ ribosomal RNA and shotgun sequencing of the whole metagenome revealed the root microbiome structures of the two tomato varieties. The rhizospheres of both varieties showed similar diversity, but the Hawaii 7996 microbiome had a higher enrichment of Flavobacteriaceae bacteria.
Transplant experiments showed that planting Moneymaker in soil where Hawaii 7996 has grown can slow down the progression of symptoms caused by R. solanacearum. Therefore, environmental factors and microbiota associated with soil might contribute to the enhanced resistance. As root exudates were shown to have no effect on the growth of $R$. solanacearum in vitro, the root microbiota probably confers the resistance to bacterial wilt.

The researchers then compared the metagenomes of rhizospheres between the resistant and susceptible varieties and thereby identified an assembled genome of a flavobacterium, termed TRM1, that has a much higher coverage in the microbiome of the resistant line than that of the susceptible line. Using the genome information of the bacterium, they designed strategies to successfully isolate and cultivate TRM1. In pot experiments, TRM1 was able to inhibit the progress of bacterial wilt in a dosage-dependent manner.

Jun Lyu

Published online: 5 December 2018 https://doi.org/10.1038/s41477-018-0335-2 